\title{
Physical Origin of Current Collapse in Au-free AlGaN/GaN Schottky Barrier Diodes
}

\author{
J. Hu ${ }^{\mathrm{a}, \mathrm{b}}$, S. Stoffels ${ }^{\mathrm{a}}, \mathrm{S}$. Lenci ${ }^{\mathrm{a}}$, N. Ronchi ${ }^{\mathrm{a}}$, R. Venegas ${ }^{\mathrm{a}}, \mathrm{S}$. You ${ }^{\mathrm{a}}$, \\ B. Bakeroot ${ }^{\mathrm{a}, \mathrm{c}}$, G. Groeseneken ${ }^{\mathrm{a}, \mathrm{b}}$, S. Decoutere ${ }^{\mathrm{a}}$ \\ ${ }^{a}$ imec vzw, Kapeldreef 75, B-3001, Heverlee, Belgium \\ Corresponding author. Phone: +32 (0) 16283275 E-mail: Jie.Hu@imec.be \\ ${ }^{\mathrm{b}}$ KU Leuven, Department of Electrical Engineering \\ Kasteelpark Arenberg 10, postbus 2440, B-3001, Heverlee, Belgium \\ ${ }^{\mathrm{c}}$ Centre for Microsystems Technology, Ghent University, Technologiepark 914a, 9052 Gent, Belgium
}

\begin{abstract}
Dynamic characterization (Pulsed $I-V$ ) on Au-free AlGaN/GaN Schottky Barrier Diodes (SBDs) has been performed to evaluate the impact of negative quiescent bias on the forward characteristics. Results show an increase of on-resistance when more negative quiescent biases are applied, and a sudden current collapse phenomenon when the quiescent bias exceeds -175 V. Furthermore, the measurements show a common signature: the total current collapse is the result of the trapping phenomena occurring around the Schottky contact corner. The trap levels of $0.5 \mathrm{eV}$ and $1.0 \mathrm{eV}$ have been characterized from current transient spectroscopy. A TCAD model with these two trap levels as donor states at the $\mathrm{Si}_{3} \mathrm{~N}_{4} / \mathrm{AlGaN}$ interface has been defined, to understand their role and explain the observed behavior of AlGaN/GaN SBDs from this dynamic measurement. We propose that trapping at deep energy levels (Trap $1=1.0 \mathrm{eV}$ ), existing at the $\mathrm{Si}_{3} \mathrm{~N}_{4} / \mathrm{AlGaN}$ interface, is responsible for the gradual current reduction observed for negative quiescent biases up to Anode-to-Cathode voltage of $-175 \mathrm{~V}$. The electron filling of the shallower traps with high density at energy level located $0.5 \mathrm{eV}$ below the conduction band starts at higher reverse biases, resulting in a strong Fermi-level pinning, which can be the cause of sudden current collapse.
\end{abstract}

Keywords: GaN, Schottky Barrier Diode (SBD), Pulsed $I-V$, current collapse, dispersion, TCAD simulation, trapping/de-trapping, GaN-on-Si.

\section{Introduction}

AlGaN/GaN Schottky Barrier Diodes (SBDs) are promising devices for high-power, high-temperature, and high-frequency applications due to their superior material properties including high channel charge density as well as large breakdown field strength $[1,2]$. To make the technology cost-effective and compete with Si-based power devices, efforts have been done to fabricate high-performance $\mathrm{AlGaN} / \mathrm{GaN}$ SBDs on large diameter $(200 \mathrm{~mm})$ GaN-on-Si wafers with Au-free CMOS-compatible process flow $[1,2]$. The simultaneous optimization of on-state and leakage of $\mathrm{AlGaN} / \mathrm{GaN}$ SBD has been reported by using anode recess combined with different techniques, i.e. GaN:C buffer [3], field plate de- sign [5], anode cleaning [4], and design of gated edge termination $[1,2]$, etc. However, the dynamic stability of GaN-based devices can be a practical issue due to trapping/de-trapping of the surface donor-like states which are commonly known to be a major source of electrons for the formation of two-dimensional electron gas (2DEG) [6]. Several authors have studied the dynamic $\mathrm{R}_{\mathrm{ON}}$ dispersion of AlGaN/GaN High Electron Mobility Transistors (HEMTs) from pulsed $I-V$ measurements, describing the variation of 2DEG density correlated with the trapping/de-trapping of surface donor states $[7,8]$.

In this paper, we investigate the role of donor states at the $\mathrm{Si}_{3} \mathrm{~N}_{4} / \mathrm{AlGaN}$ interface on the dynamic behavior of Au-free AlGaN/GaN SBDs in pulsed regime. A simple model has been defined in the TCAD simulator, with two discrete donor trap levels at $0.5 \mathrm{eV}$ and $1.0 \mathrm{eV}$ (at the $\mathrm{Si}_{3} \mathrm{~N}_{4} / \mathrm{AlGaN}$ interface) based on the results from current transient measurements. From the simulation results, we are able to qualitatively explain the experimental observations: gradual current reduction and sudden current collapse.

\section{Device fabrication}

$\mathrm{AlGaN} / \mathrm{GaN}$ epitaxial layers were grown on 8-inch GaN-on-Si wafers by using Metalorganic Chemical Vapor Deposition (MOCVD). The epi-structure was composed by a $10 \mathrm{~nm} \mathrm{Al}_{0.25} \mathrm{Ga}_{0.75} \mathrm{~N}$ barrier, a $150 \mathrm{~nm}$ GaN channel layer, a buffer layer $\left(400 \mathrm{~nm} \quad \mathrm{Al}_{0.74} \mathrm{Ga}_{0.26} \mathrm{~N} / 400 \mathrm{~nm}\right.$ $\mathrm{Al}_{0.44} \mathrm{Ga}_{0.56} \mathrm{~N} / 1800 \mathrm{~nm} \mathrm{Al}_{0.21} \mathrm{Ga}_{0.79} \mathrm{~N}$ ), and a $200 \mathrm{~nm} \mathrm{AlN}$ nucleation layer on $p$-type $\mathrm{Si}$ (111) substrate. The surface of the entire stack was passivated by $140 \mathrm{~nm} \mathrm{Si}_{3} \mathrm{~N}_{4}$ layer by

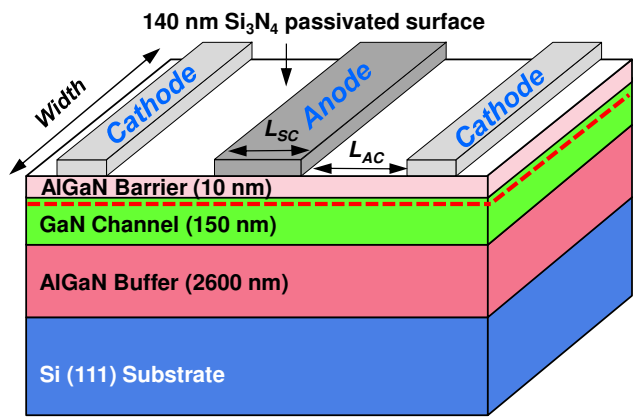

Fig. 1. Schematic structure of $\mathrm{AlGaN} / \mathrm{GaN}$-on-Si SBD with Anode-to-Cathode distance $L_{A C}=5 \mu \mathrm{m}$, Anode finger width $=100 \mu \mathrm{m}$, Schottky contact length $L_{S C}=9 \mu \mathrm{m}$. 
means of Rapid Thermal Chemical Vapor Deposition (RTCVD). The active areas were isolated by a nitrogen-based implantation. The removal of the $\mathrm{Si}_{3} \mathrm{~N}_{4}$ at the anode region was performed with a $\mathrm{SF}_{6}$ dry etch. Before the deposition of the Anode metal, a $\mathrm{N}_{2}$ plasma treatment at the Anode area was introduced to remove the native oxides or traps located at the Schottky junction for the improvements in performance $[9,10]$. The $\mathrm{AlGaN} / \mathrm{GaN}$ SBDs were then fabricated with $\mathrm{Au}$-free Anode and Cathode contacts [1, 2]. The schematic of the SBDs studied is shown in Fig. 1 with symmetric configuration. The devices measured in this work are single anode finger AlGaN/GaN SBDs with the following layout parameters: Anode-to-Cathode distance $L_{A C}=5 \mu \mathrm{m}$, Anode finger width $=100 \mu \mathrm{m}$, and length of Schottky contact $L_{S C}=9 \mu \mathrm{m}$.

\section{Experiment and simulation}

To examine the dynamic behavior of AlGaN/GaN SBDs, we developed a measurement procedure shown in Fig. 2 and used an Agilent B1505A to measure the pulsed $I-V$ characteristics. Each point of the forward $I-V$ characteristics is preceded by a $10 \mathrm{~ms}$ stressing pulse (quiescent state) at a negative bias $\left(V_{R}\right)$. During the filling pulse, the high electric field changes the state of the surface donors at the corner of the Schottky contact (where the electric field has its intensity peak) [2]. The forward behavior is then affected by the trapping phenomena occurred during the filling pulse. The pulsed forward characteristic has been investigated for different stressing voltages $V_{R}\left(V_{R}\right.$ from $0 \mathrm{~V}$ to $-200 \mathrm{~V})$ to understand the trapping mechanisms.

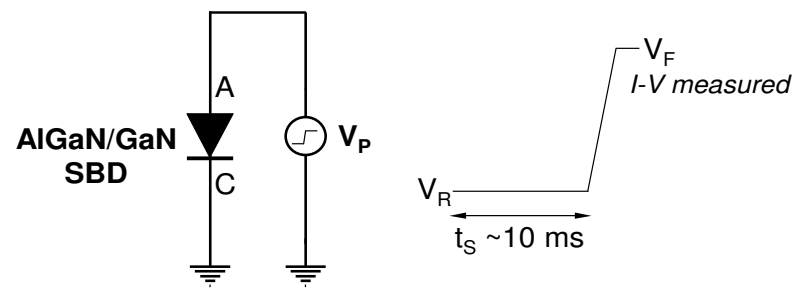

Fig. 2. Experimental set-up for Pulsed $I-V$ measurement on AlGaN/GaN SBD (left); Measurement technique (right): forward current was measured with a pulsed positive voltage $V_{F}$ before when a constant stressing voltage $V_{R}$ was applied on the Anode with stressing time period ts of 10 ms.

A two-dimensional TCAD simulation has been carried out using the commercial software Synopsys Sentaurus Device. Donor-like trap levels have been defined at the $\mathrm{Si}_{3} \mathrm{~N}_{4} / \mathrm{AlGaN}$ interface with a uniform spatial distribution in density. The traps were defined as bulk traps located in a small region close to AlGaN surface $(0.5 \mathrm{~nm})$ which allows for a clearer visualization of the trap occupation under different bias conditions. There is no trap states defined at the Anode metal/AlGaN barrier interface. The densities of the donor traps are set as $1.25 \times 10^{12} \mathrm{~cm}^{-2}$ and $2.1 \times 10^{13} \mathrm{~cm}^{-2}$ at "Trap1" and "Trap2" with their energy levels extracted from the current transient measurements at different temperatures. As shown in Fig. 3 (left), the Fermi-level is lo-
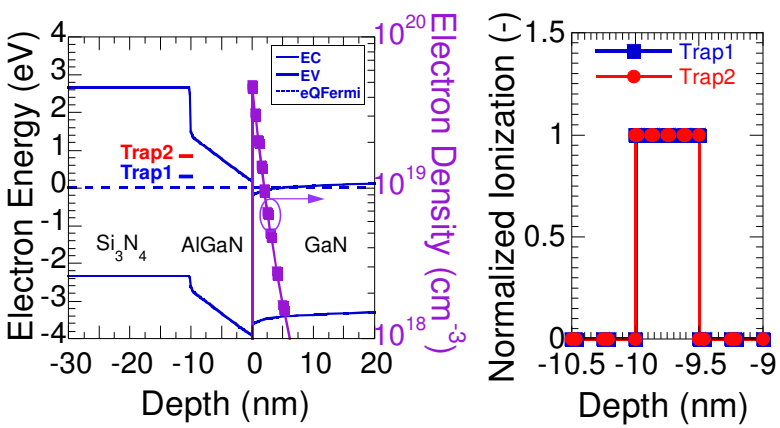

Fig. 3. The simulated band diagram and $2 \mathrm{DEG}$ density at equilibrium (left), and normalized ionization of the traps defined at the $\mathrm{Si}_{3} \mathrm{~N}_{4} / \mathrm{AlGaN}$ interface (right).

cated below the donor trap levels at the $\mathrm{Si}_{3} \mathrm{~N}_{4} / \mathrm{AlGaN}$ interface at equilibrium state with $\mathrm{V}_{\mathrm{AC}}=0 \mathrm{~V}$. Donor trap carries a positive charge when it is ionized and stays neutral when it is occupied with an electron. The normalized ionization of the two traps defined is shown in Fig. 3 (right) to be "1" [11], i.e. all the interface traps are ionized with positive charges which compensates the negative polarization charges at the $\mathrm{AlGaN}$ surface and gives rise to a high 2DEG density in the channel shown to the right axis in Fig. 3 (left).

\section{Results and discussion}

In Fig. 4, typical pulsed $I-V$ characteristics with changing $V_{R}$ for $A l G a N / G a N ~ S B D$ are shown. Two regimes have been observed in terms of current levels: gradual current reduction before a critical voltage and total current collapse when $V_{R}$ goes beyond the critical voltage. Before reaching the critical voltage of $V_{R}=-175 \mathrm{~V}$ in this measurement, the diode can still turn on around $1.5 \mathrm{~V}$ (with criteria of $\mathrm{I}_{\mathrm{AC}}=$ $1 \mathrm{~mA} / \mathrm{mm}$ after normalization of the current to the width of Anode finger). It is clearly observed that there is a gradual reduction of maximal forward current at $\mathrm{V}_{\mathrm{F}}$ of $2.5 \mathrm{~V}$ by increasing the reverse bias from $0 \mathrm{~V}$ to $-150 \mathrm{~V}$, which is correlated with the increase of on-resistance as calculated in Fig. 4 (right). Moreover, the totally collapsed current can still abruptly jump to the "normal" level when a sufficiently high forward voltage triggers the de-pinning of the Fermi level.
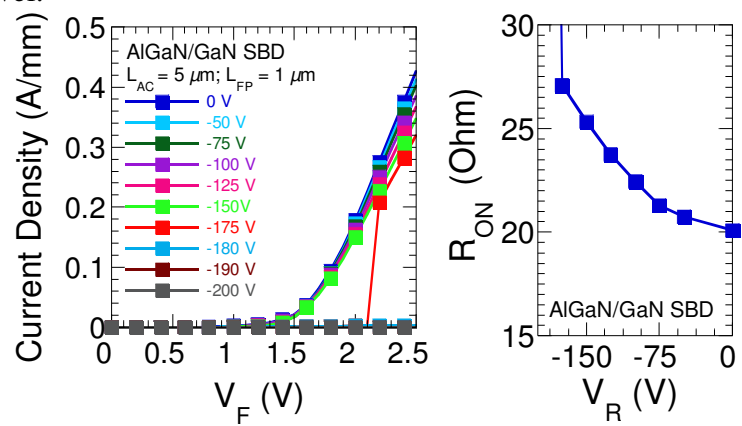

Fig. 4. Pulsed forward $I-V$ characteristics with a variation of the stressing voltage $V_{R}$ from $0 \mathrm{~V}$ to $-200 \mathrm{~V}$ (left); The variation of on-resistance with changing $V_{R}$ which correlates to the current reduction (right). 
During the off-state operation, there is an electric field peak located at the corner of Schottky contact which results in a perimeter effect of leakage current [2]. It is important to note that there is a peak lateral component of the electric field which attracts electrons to be captured by the traps present at the $\mathrm{Si}_{3} \mathrm{~N}_{4} / \mathrm{AlGaN}$ interface. Electron trapping is responsible for this current-reduction and collapse, as the trapped charges act as a virtual gate which deplete the 2DEG channel existing underneath when the forward current is measured [12].

From the observation in Fig. 4 (left), there are two distinct traps which are responsible for the gradual current reduction and total current collapse, respectively. Current transient measurement after stressing the AlGaN/GaN SBDs has been performed to identify the trap energy levels. As can be shown in Fig. 5, thermally activated energy levels of $\sim 1.0 \mathrm{eV}$ (Trap1) and $0.5 \mathrm{eV}$ (Trap2) are evaluated. The trap states can be due to the threading dislocations [13] and AlGaN surface states [14].
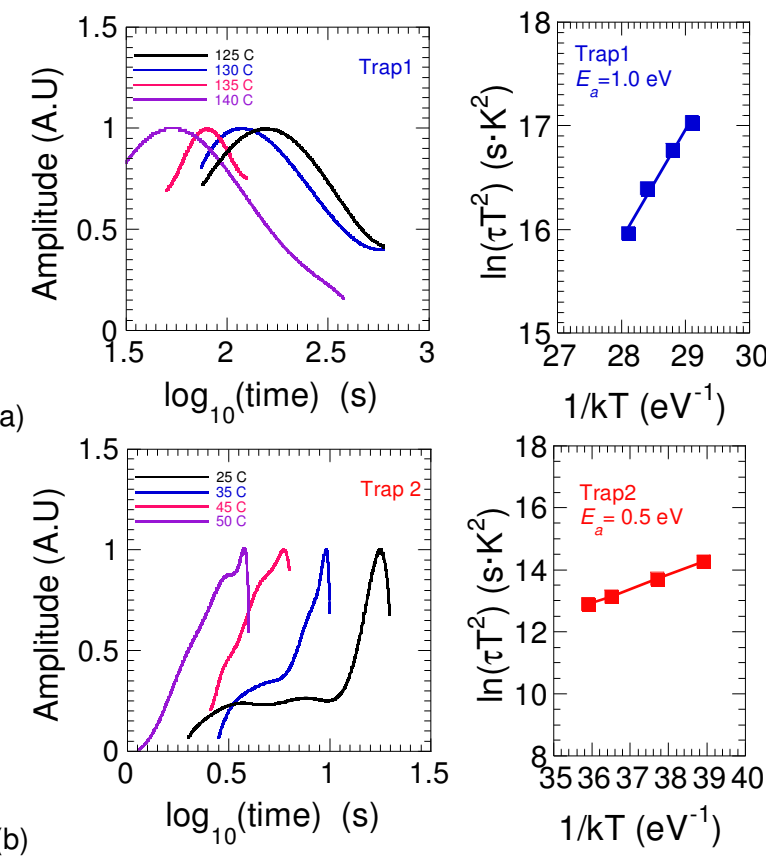

Fig. 5. Time constant spectra (derivative of the current transients) measured at different temperatures and the Arrhenius plots for Trap1 (a) and Trap2 (b), respectively.

At low voltage range of $V_{R}$, the electrons are firstly captured by "Trap1" due to their lower energy as shown in Fig. 6 (left). As the increase of negative bias, there is a rise of the peak lateral electric field which results in more electron trapping in the vicinity of Schottky contact. The simulated band diagram (at a distance of $0.5 \mathrm{~nm}$ from the Anode metal) at $\mathrm{V}_{\mathrm{AC}}=-150 \mathrm{~V}$ is shown in Fig . 6 (right). It is illustrated in the figure that the quasi-Fermi level is pinned at the Trap1 energy level with electrons occupying those deeper trap states. The Trap2 is still located above the quasi-Fermi level and remains ionized. Due to the relatively low density of Trap1, the 2DEG channel can still be formed when a pulsed forward voltage is applied.
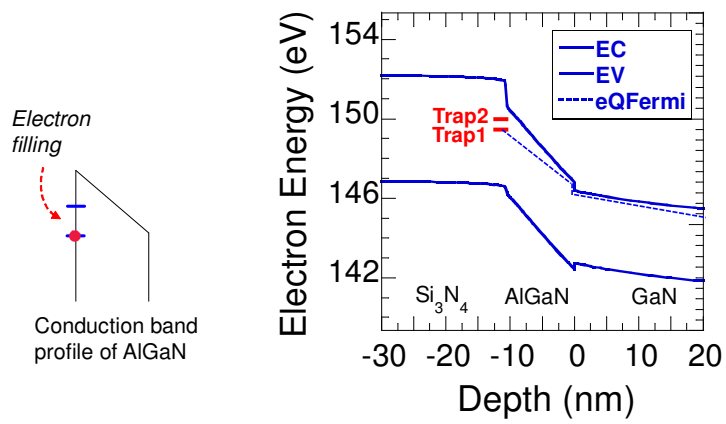

Fig. 6. The conduction band profile of AlGaN barrier layer under reserve biased conditions when the injected electron is captured by a deep trap level (left); The simulated band diagram at $\mathrm{V}_{\mathrm{AC}}=-150 \mathrm{~V}$ (right).

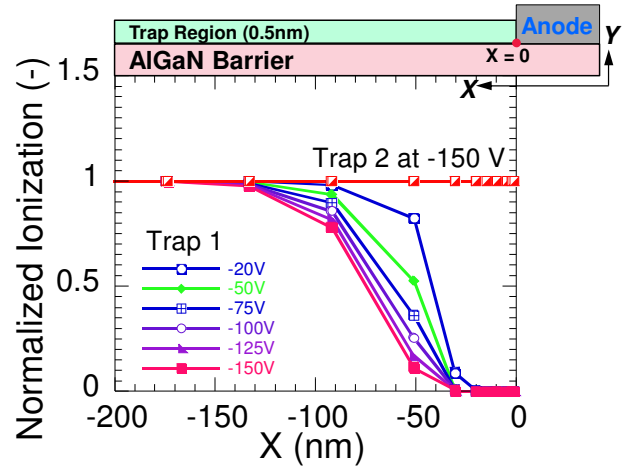

Fig. 7. The lateral distribution of normalized ionization for "Trap1" with different $V_{R}$ down to $-150 \mathrm{~V}$ and for "Trap2" at $\mathrm{V}_{\mathrm{R}}=-150 \mathrm{~V}$. The position of $\mathrm{X}=0 \mathrm{~nm}$ corresponds to the corner of Schottky contact.

TCAD simulation results obtained using trap occupation described in Fig. 7 have verified that the trapping phenomena were occurred at the corner of the Schottky contact, and a lateral extension of trapped states for "Trap1" were observed when reverse bias becomes more negative. The occupied donor states induces a field effect which reduces the density of 2DEG resulting in a higher on-resistance as observed from experimental data shown in Fig. 4 (right). The "Trap2" stays ionized until $\mathrm{V}_{\mathrm{AC}}=-150 \mathrm{~V}$.

At even more negative bias, "Trap2" starts to be filled with injected electrons due to the further bending of the band laterally. The simulated band diagram (at $\mathrm{V}_{\mathrm{AC}}=-175$ V) in Fig. 8 (left) shows that the quasi-Fermi level at the $\mathrm{Si}_{3} \mathrm{~N}_{4} / \mathrm{AlGaN}$ interface is pinned at the trap level of $0.5 \mathrm{eV}$, which demonstrates the charging effect at the energy level of "Trap2". The normalized ionization from simulation in Fig. 8 (right) confirms the process of electron-filling at "Trap2" at more negative bias (the simulated $\mathrm{V}_{\mathrm{AC}}=-175$ $\mathrm{V})$, since the normalized ionization of "Trap2" starts to drop. Due to the large trap density at $0.5 \mathrm{eV}$, the current can be blocked at the forward regime before the discharging of these trapped states and de-pinning of the Fermi-level take place. 

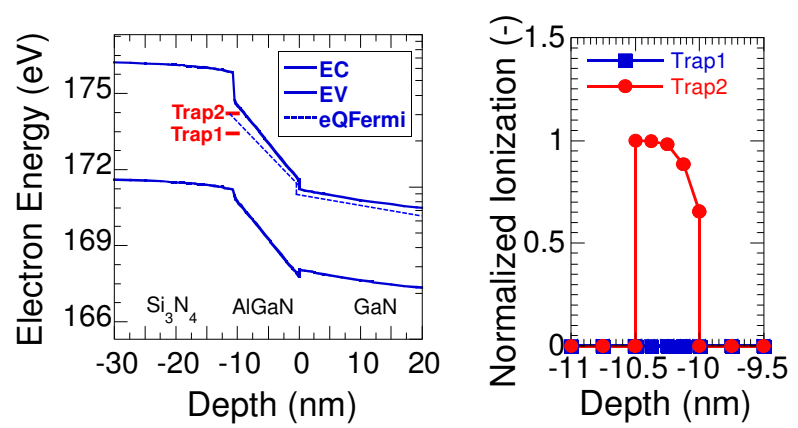

Fig. 8. The simulated band diagram at $\mathrm{V}_{\mathrm{AC}}=-175 \mathrm{~V}$ for SBD (right), the normalized ionization of "Trap1" and "Trap2" at $\mathrm{V}_{\mathrm{AC}}=-175 \mathrm{~V}$.

From the measurement results shown in Fig. 4, there is a jump of current when the device was stressed with $V_{R}$ of $-175 \mathrm{~V}$. This is a signature of de-pinning of the Fermi-level at the $\mathrm{Si}_{3} \mathrm{~N}_{4} / \mathrm{AlGaN}$ interface with a distance of several nanometers from the corner of Schottky contact where a positive pulse voltage was applied. Most importantly, this phenomenon of Fermi-level de-pinning can only take place with limited distance away from the Anode metal, since it is triggered by a "small" positive voltage.

It is worth mentioning that more trap levels can be involved during this trapping process at off-state. However, we have demonstrated the contribution of different trap levels at different bias conditions and given qualitative explanation to the experimental observations. This paper also indicates that mitigation of the current collapse of AlGaN/GaN SBDs can be realized by improving the $\mathrm{Si}_{3} \mathrm{~N}_{4} / \mathrm{AlGaN}$ interface properties.

\section{Conclusions}

This work correlates results of pulsed $I-V$ experimental observations on Au-free AlGaN/GaN Schottky Barrier Diodes with trapping phenomena of surface donor states. A simple model was implemented in a two-dimensional numerical simulator which can explain the measurement results. In this model, two trap levels were defined with different behaviors. The gradual on-resistance increase and total current collapse were related to the electron-filling of these trap states which depends on the applied stressing bias. By analyzing the trapping occupation of different donor trap levels at different voltage regions, we are able to verify the impact of the surface donor states on the dynamic behavior of $\mathrm{AlGaN} / \mathrm{GaN}$ SBDs.

\section{Acknowledgements}

This work was performed with support from imec IIAP program. The authors would like to acknowledge the efforts of Epi-team for wafer growth and fruitful discussions with partners.

\section{References}

[1] S. Lenci, B. De Jaeger, L. Carbonell, et al., "Au-Free $\mathrm{AlGaN} / \mathrm{GaN}$ power diode on 8-in $\mathrm{Si}$ substrate with gated edge termination," IEEE Electron Device Lett., vol. 34, no.8, pp. 1035-1037, Aug. 2013.

[2] J. Hu, S. Lenci, S. Stoffels, et al., "Leakage-current reduction and improved on-state performance of $\mathrm{Au}$-free AlGaN/GaN-on-Si Schottky diode by embedding the edge terminations in the anode region," Phys. Status Solidi C, no. 3-4, pp. 862-865, Jan. 2014.

[3] E. Bahat-Treidel, O. Hilt, R. Zhytnytska, et al., "Fast-switching GaN based lateral power Schottky barrier diodes with low onset voltage and strong reverse blocking," IEEE Electron Device Lett., vol. 33, no. 3, pp. 357-359, Mar. 2012.

[4] J.J.T.M. Donkers, S.B.S. Heil, G.A.M. Hurkx, et al., "600V-900V GaN-on-Si process technology for Schottky Barrier Diodes and power switches fabricated in a standard Si-production fab," CS MANTECH Conference, pp. 259-262, May 2013.

[5] T. Boles, C. Varmazis, D. Carlson, et al., "High-Voltage GaN-on-Silicon Schottky Diodes", CS MANTECH Conference, pp. 297-300, May 2013.

[6] J. B. Ibbetson, P. T. Fini, K. D. Ness, et al., "Polarization effects, surface states, and the source of electrons in AlGaN/GaN heterostructure field effect transistor," Appl. Phys. Lett., vol. 77, no. 2, pp. 250-252, Jul. 2000.

[7] G. Meneghesso, G. Verzellesi, R. Pierobon, et al., "Surface-related drain current dispersion effects in AlGaN-GaN HEMTs," IEEE Trans. Electron Devices, vol. 51, no. 10, pp. 1554-1561, Oct. 2004.

[8] G. Longobardi, F. Udrea, S. Sque, et al., "Impact of donor traps on the 2DEG and electrical behavior of $\mathrm{AlGaN} / \mathrm{GaN}$ MISFETs," IEEE Electron Device Lett., vol. 35, no.1, pp. 27-29, Jan. 2014.

[9] J.H. Kim, H.G. Choi, M-W Ha, et al., "Effects of nitride-based plasma pretreatment prior to SiNx passivation in $\mathrm{AlGaN} / \mathrm{GaN}$ High-Electron-Mobility Transistors on silicon substrates", Jpn. J. Appl. Phys. vol. 49, p. 04DF05, 2010.

[10] D.J. Meyer, J.R. Flemish, and J.M. Redwing, "Plasma surface pretreatment effects on silicon nitride passivation of AlGaN/GaN HEMTs," in Proc. CS MANTECH, pp. 305-307, May 2007.

[11] Device simulator Sentaurus device, version G-2012.06, Synopsys Inc., 2012.

[12] R. Vetury, N. Q. Zhang, S. Keller, et al., "The impact of surface states on the DC and RF characteristics of AlGaN/GaN HFETs," IEEE Trans. Electron Devices, vol. 48, no. 3, pp. 560-566, Mar. 2001.

[13] Z. Q. Fang, D. C. Look, D. H. Kim, et al., Appl. Phys. Lett., 87, 182115, Oct. 2005.

[14] A. R. Arehart, A. Sasikumar, S. Rajan, et al., Solid-State Electron. 80, pp. 19-22, Nov. 2012. 